\title{
Self-Learning Method for Construction of Analytical Interatomic Potentials to Describe Laser-Excited Materials
}

\author{
Bernd Bauerhenne ${ }^{1},{ }^{*}$ Vladimir P. Lipp $\odot,{ }^{1,2}$ Tobias Zier®, ${ }^{1}$ Eeuwe S. Zijlstra, ${ }^{1}$ and Martin E. Garcia ${ }^{1}$ \\ ${ }^{1}$ Theoretical Physics and Center for Interdisciplinary Nanostructure Science and Technology (CINSaT), \\ University of Kassel, Heinrich-Plett-Straße 40, 34132 Kassel, Germany \\ ${ }^{2}$ Center for Free-Electron Laser Science CFEL, Deutsches Elektronen-Synchrotron DESY, 22607 Hamburg, Germany
}

(Received 21 December 2018; revised manuscript received 19 December 2019; accepted 14 January 2020; published 25 February 2020)

\begin{abstract}
Large-scale simulations using interatomic potentials provide deep insight into the processes occurring in solids subject to external perturbations. The atomistic description of laser-induced ultrafast nonthermal phenomena, however, constitutes a particularly difficult case and has so far not been possible on experimentally accessible length scales and timescales because of two main reasons: (i) ab initio simulations are restricted to a very small number of atoms and ultrashort times and (ii) simulations relying on electronic temperature- $\left(T_{e}\right)$ dependent interatomic potentials do not reach the necessary ab initio accuracy. Here we develop a self-learning method for constructing $T_{e}$-dependent interatomic potentials which permit ultralarge-scale atomistic simulations of systems suddenly brought to extreme nonthermal states with density-functional theory (DFT) accuracy. The method always finds the global minimum in the parameter space. We derive a highly accurate analytical $T_{e}$-dependent interatomic potential $\Phi\left(T_{e}\right)$ for silicon that yields a remarkably good description of laser-excited and -unexcited Si bulk and Si films. Using $\Phi\left(T_{e}\right)$ we simulate the laser excitation of Si nanoparticles and find strong damping of their breathing modes due to nonthermal melting.
\end{abstract}

DOI: 10.1103/PhysRevLett.124.085501

Intense femtosecond-laser pulses can induce dramatic changes in the potential energy surface (PES) of materials due to the excitation of a large amount of electron-hole pairs [1]. These changes manifest themselves in the form of bond hardening, bond softening, and lattice instabilities, for example [2-4]. It is known that these light-induced effects lead to measurable ultrafast nonthermal phenomena such as structural solid-solid and solid-liquid phase transitions [5-7], phonon squeezing [8,9], and coherent phonons $[10,11]$. However, the impact of short-lived changes in the PES, occurring within the first picosecond after an ultrashort excitation [12], on the further dynamics of the material at longer timescales (nanoseconds) and on larger length scales (hundreds of nanometers) still remains unknown. As an example, it has been proposed that bond softening and instabilities occurring immediately after the action of a femtosecond-laser pulse followed by a relaxation involving self-organization processes might play a role in the spontaneous formation of surface patterns like laser-induced periodic surface structures [13-15]. However, this hypothesis could not be theoretically tested so far because of the lack of an ab initio description of laserinduced nonthermal material transformations on macroscopic length scales and long timescales, which constitutes a still-unresolved problem in the theory of ultrafast phenomena. This is due to the fact that ab initio molecular dynamics (MD) simulations are typically restricted to, at most, 1000 atoms $[9,16,17]$ whereas, on the other hand, large-scale MD simulations based on interatomic potentials depending on the electronic temperature $T_{e}$, i.e., taking laser-induced changes of the PES into account [18-23], have not been so far able to achieve ab initio accuracy.

In this Letter, we develop a self-learning method for deriving analytical $T_{e}$-dependent interatomic potentials that have a physically intuitive form and reproduce the results of $a b$ initio MD simulations with minimal error and computational effort. As an example, we applied this method to $\mathrm{Si}$ due to its technological importance. Another motivation to derive $\Phi\left(T_{e}\right)$ for $\mathrm{Si}$ is that it can be directly compared with other available $T_{e}$-dependent $\mathrm{Si}$ potentials $[19,23]$, which permits us to appreciate the high accuracy of our derived potential. Moreover, laser-excited Si has been intensively studied by means of ab initio calculations [2,9,16,17, 24-27]. As a first application of $\Phi\left(T_{e}\right)$, we predict, by means of atomistic simulations, a strongly damped breathing mode in laser-excited spherical Si nanoparticles.

To derive $\Phi\left(T_{e}\right)$, we must take into account that, ideally, it should reproduce forces and energies over any pathway on the $a b$ initio PES. In practice, we perform reference $a b$ initio simulations on a treatable small cell and require that $\Phi\left(T_{e}\right)$ matches forces and energies at every simulation step $t$. The reference simulations were performed on a cell consisting of $320 \mathrm{Si}$ atoms forming a thin film of $5.3 \mathrm{~nm}$ thickness to take both bulk and surface effects into account. The construction of a reliable interatomic potential needs a 
very good sampling of the available phase space. In case of laser excitation, it should include extreme fluctuations of local atomic configurations. For this reason, we performed various sets of reference $a b$ initio simulations (labeled as $\left.s_{k}\right)$ as follows [28]. $\left(s_{1}\right)$ We simulated the laser-induced dynamics of a thin film-initially thermalized at an ionic temperature, $T_{i}=T_{e}=1 \mathrm{mHa}(316 \mathrm{~K})$-by suddenly increasing $T_{e}$ to a constant value. We considered 11 different $T_{e}$ 's in the range $1 \mathrm{mHa}(316 \mathrm{~K})-100 \mathrm{mHa}$ $(31577 \mathrm{~K})$ [28]. $\left(s_{2}\right)$ Since the laser excitation of solids can lead to high local positive pressures with high atomic local densities, we compressed the thin film initialized at $1 \mathrm{mHa}$ $(316 \mathrm{~K})$ stepwise and calculated the related forces and energies. $\left(s_{3}\right)$ Since the laser excitation of solids can also lead to high local negative pressures with low local atomic densities, the $a b$ initio calculations must also contain a mechanical expansion of the thin film for each studied $T_{e}$. Note that expansion occurs naturally for high $T_{e}$ 's in simulation set $s_{1}$. For low $T_{e}$ 's we manually simulated expansion [28]. In total, we obtained around $10^{6}$ ab initio data points for low $T_{e}$ 's and $5 \times 10^{5}$ for high $T_{e}$ 's.

The $a b$ initio simulations were performed using our inhouse density-functional theory (DFT) program CHIVES (Code for Highly excIted Valence Electron Systems) $[3,9,17]$ within the local density approximation (LDA). CHIVES reproduces results of established DFT codes, including Wien2k and ABINIT [33], yields a very good agreement with experiment [34], and is up to 200 times faster than ABINIT with the same degree of accuracy [33].

To obtain a reliable and appealing analytical form of $\Phi\left(T_{e}\right)$, we construct it as a sum of different local interaction terms with a clear physical interpretation. We use two- and three-body interaction terms $\left(\Phi_{2}\right.$ and $\Phi_{3}$, respectively) to account for covalent bonding. Note that two- and threebody terms were already successfully applied to describe the Si crystal in the electronic ground state [35], where covalent bonds are responsible for the tetragonal bonding geometry. In addition, we account for metallic bonding, which dominates in Si under pressure [36] or in the molten phase [16], by using an embedding function $\Phi_{\rho}$. Here, the local potential energy at an atomic site is calculated as a function of the surrounding atomic density $\rho$. Embedding functions were already successfully applied for describing metals [37,38]. $\Phi\left(T_{e}\right)$ reads

$$
\begin{aligned}
\Phi= & \sum_{\substack{i<j \\
r_{i j}<r_{2}^{(c)}}} \Phi_{2}\left(T_{e}, r_{i j}\right)+\sum_{\substack{i j k \\
r_{i j}, r_{i k}<r_{3}^{(c)}}}^{\prime} \Phi_{3}\left(T_{e}, r_{i j}, r_{i k}, \theta_{i j k}\right) \\
& +\sum_{i} \Phi_{\rho}\left(T_{e}, \rho_{i}^{(2)}, \rho_{i}^{(3)}, \ldots, \rho_{i}^{\left(N_{\rho}^{(r)}\right)}\right)+\sum_{i} \Phi_{0}\left(T_{e}\right) .
\end{aligned}
$$

Here $r_{i j}$ denotes the distance between atoms $i$ and $j, \theta_{i j k}$ is the angle between $\mathbf{r}_{i j}$ and $\mathbf{r}_{i k}$, the prime indicates that all summation indices are distinct, and $\rho_{i}^{(2)}, \rho_{i}^{(3)}, \ldots$ are different measures for the atomic density surrounding atom $i$ (see below). $\Phi_{0}\left(T_{e}\right)$ is the Helmholtz free energy of an isolated atom, which is needed to reproduce correctly the derivatives of $\Phi\left(T_{e}\right)$ with respect to $T_{e}$.

In Eq. (1), $r_{2}^{(c)}, r_{3}^{(c)}$, and $r_{\rho}^{(c)}$ (see below) denote the individual cutoff radii for $\Phi_{2}, \Phi_{3}$, and $\Phi_{\rho}$, respectively. The cutoff radius is defined as the distance beyond which the interaction between atoms is set equal to zero and, therefore, it controls the interaction range of the corresponding term. For covalent and metallic bonding, the interatomic interactions become negligible at relatively short interatomic distances, as can be seen for $\mathrm{Si}$ from the $a b$ initio data in the bottom inset of Fig. 2. Therefore, a physical upper limit exists for the cutoff radii in such systems. By discretely testing all values of the cutoff radii starting from the nearest neighbor distance occurring in the equilibrium crystal structure up to the previously mentioned upper limit, we surely find the optimal cutoff radii for $\Phi\left(T_{e}\right)$ [28]. For ionic systems with long-ranged Coulomb interaction, this procedure must be modified.

Our goal is to construct an expression for $\Phi\left(T_{e}\right)$ that is very flexible and simple enough to allow the optimization procedure to find the global minimum in the parameter space. Therefore, we expand the terms $\Phi_{2}, \Phi_{3}$, and $\Phi_{\rho}$ into polynomials, which can, in principle, reproduce any physically reasonable function. $\Phi_{2}, \Phi_{3}$, and $\Phi_{\rho}$ are constructed as

$$
\begin{gathered}
\Phi_{2}=\sum_{q=2}^{N_{2}^{(r)}} c_{2}^{(q)}\left(1-\frac{r_{i j}}{r_{2}^{(c)}}\right)^{q} \\
\Phi_{3}=\sum_{q_{1}=2}^{N_{3}^{(r)}} \sum_{q_{2}=q_{1}}^{N_{3}^{(r)}} \sum_{q_{3}=0}^{N_{3}^{(\theta)}} c_{3}^{\left(q_{1} q_{2} q_{3}\right)} \\
\times\left(1-\frac{r_{i j}}{r_{3}^{(c)}}\right)^{q_{1}}\left(1-\frac{r_{i k}}{r_{3}^{(c)}}\right)^{q_{2}}\left[\cos \left(\theta_{i j k}\right)\right]^{q_{3}}, \\
\Phi_{\rho}=\sum_{q_{1}=2}^{N_{\rho}^{(r)}} \sum_{q_{2}=1}^{N_{\rho}^{(\rho)}} c_{\rho}^{\left(q_{1} q_{2}\right)}\left(\frac{\rho_{i}^{\left(q_{1}\right)}}{1+\rho_{i}^{\left(q_{1}\right)}}\right)^{q_{2}},
\end{gathered}
$$

and, for $q_{1}=2,3, \ldots, N_{\rho}^{(r)}$, the measures for the atomic density surrounding atom $i$ are constructed as

$$
\rho_{i}^{\left(q_{1}\right)}=\sum_{\substack{j \neq i \\ r_{i j}<r_{\rho}^{(c)}}}\left(1-\frac{r_{i j}}{r_{\rho}^{(c)}}\right)^{q_{1}} .
$$

The expansions (2)-(4) guarantee high numerical stability and that the interatomic potential and its first derivatives decrease continuously to zero as distances reach the cutoff radii [28]. 
To determine the accuracy of $\Phi\left(T_{e}\right)$ in reproducing the $a b$ initio reference simulations $\left\{s_{k}\right\}$ at a given $T_{e}$, we defined the following error function $W\left(T_{e}\right)$ (cf. Ref. [39]), which is the weighted sum of the mean square relative errors in the $\Phi\left(T_{e}\right)$ description of Helmholtz free cohesive energies and atomic forces of the different simulations $\left\{s_{k}\right\}$ with weights $w_{E}^{\left(s_{k}\right)}, w_{f}^{\left(s_{k}\right)}$, respectively, obeying the sum rule $\sum_{s_{k}} w_{E}^{\left(s_{k}\right)}+\sum_{s_{k}} w_{f}^{\left(s_{k}\right)}=1$ :

$$
\begin{aligned}
W= & \sum_{s_{k}} w_{E}^{\left(s_{k}\right)} \frac{\sum_{t}\left[\Phi\left(\left\{\mathbf{r}_{j}^{\left(s_{k}\right)}(t)\right\}\right)-\sum_{i} \Phi_{0}-E^{\left(s_{k}\right)}(t)\right]^{2}}{\sum_{t}\left[E^{\left(s_{k}\right)}(t)\right]^{2}} \\
& +\sum_{s_{k}} w_{f}^{\left(s_{k}\right)} \frac{\sum_{t i}\left|-\nabla_{\mathbf{r}_{i}^{\left(s_{k}\right)}} \Phi\left(\left\{\mathbf{r}_{j}^{\left(s_{k}\right)}(t)\right\}\right)-\mathbf{f}_{i}^{\left(s_{k}\right)}(t)\right|^{2}}{\sum_{t i}\left|\mathbf{f}_{i}^{\left(s_{k}\right)}(t)\right|^{2}} .
\end{aligned}
$$

Here, $\mathbf{r}_{i}^{\left(s_{k}\right)}$ denotes the position of atom $i, E^{\left(s_{k}\right)}(t)$ the total $a b$ initio Helmholtz free cohesive energy of the system, and $\mathbf{f}_{i}^{\left(s_{k}\right)}(t)$ the ab initio force acting on atom $i$. Since we aim at describing all considered $T_{e}$ 's with a minimal error, we finally use the average of $W\left(T_{e}\right)$ over $T_{e}=316 \mathrm{~K}$ and $T_{e}=18946 \mathrm{~K}$ as a measure of the fitting error, which we denote as $\langle W\rangle$.

The polynomial degrees $N_{2}^{(r)}, N_{3}^{(r)}, N_{3}^{(\theta)}, N_{\rho}^{(r)}$, and $N_{\rho}^{(\rho)}$ control the complexity of the different local interaction terms $\Phi_{2}, \Phi_{3}$, and $\Phi_{\rho}$. By adjusting the polynomial degrees, we can adapt the analytical form of the interatomic potential to reproduce the $a b$ initio data most accurately and efficiently. For this, we require minimal error $\langle W\rangle$, and, at the same time, reasonably low computational cost, i.e., a small total number of coefficients $N_{c}$. In principle, for an optimal selection of the final polynomial-degree combination, we would have to consider an infinite number of possible degree combinations. Fortunately, the polynomial expansion of Eqs. (2)-(4) converges to the ab initio determined PES relatively quickly, so that the inclusion of high degrees does not bring significant improvement in accuracy, as can be seen in Fig. 1. Consequently, high degrees can be excluded. Therefore, we initially set arbitrary upper degree limits and determined the minimal error $\langle W\rangle$ for all polynomial-degree combinations up to these limits. From the corresponding calculations, we can check whether the initially chosen upper degree limits were high enough (see Fig. 1) and can eventually increase them. In this way, we perform a complete search over all "reasonable" interatomic potentials of the form of Eq. (1). Moreover, due to the construction of the interatomic potential, one can reliably calculate the global minimum of $\langle W\rangle$ and the corresponding optimal parameters $\left(N_{c}\right.$ coefficients and 3 cutoff radii) for all considered polynomial-degree combinations, since all coefficients $\left\{c_{2}^{(q)}\right\}$,

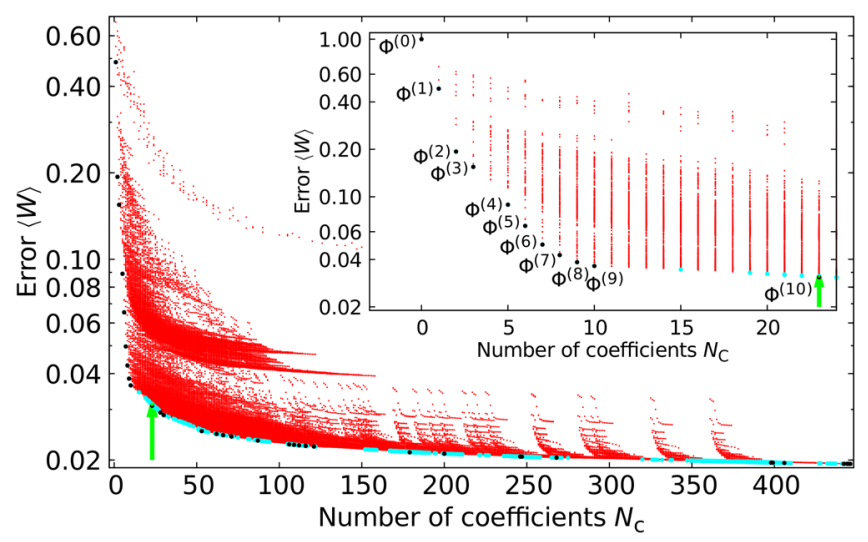

FIG. 1. Error $\langle W\rangle$ averaged over $T_{e}=316 \mathrm{~K}$ and $T_{e}=$ $18946 \mathrm{~K}$ as a function of $N_{c}$ for all polynomial-degree combinations up to the upper degree limits (see text). Each red dot represents an interatomic potential which is, for the corresponding polynomial-degree combination, a global minimum in parameter space. $\langle W\rangle$ decreases insignificantly for large values of $N_{c}$, indicating that the chosen upper degree limits are high enough. The subset $S_{\text {best }}$ is highlighted in light blue (see text), the series $\Phi^{(k)}$ is highlighted in black (see text), and the final choice, $\Phi\left(T_{e}\right)$, is highlighted in green and marked by an arrow.

$\left\{c_{3}^{\left(q_{1} q_{2} q_{3}\right)}\right\}$, and $\left\{c_{\rho}^{\left(q_{1} q_{2}\right)}\right\}$ are independent from each other and enter linearly into the potential [see Eqs. (2)-(4)] [28]. The ability to determine the global minimum of $\langle W\rangle$ allows us to find, for any polynomial-degree combination (i.e., for any analytical form of the interatomic potential) the related parameters that describe the ab initio data most accurately.

Figure 1 presents the global minimum of $\langle W\rangle$ (red points) as a function of the total number of coefficients $N_{c}$ for 165344 polynomial-degree combinations, which we obtained by taking all polynomial degrees into account up to the upper degree limits $N_{2}^{(r, \max )}=15$ for the two-body term and $N^{(\max )}=9$ for all other terms.

To select the polynomial-degree combination of the final interatomic potential showing physical behavior and having minimal $\langle W\rangle$ and reasonably low computational cost, we first extracted a relatively small subset $S_{\text {best }}$ of reliable polynomial-degree combinations that exhibit low $\langle W\rangle$ combined with a relatively small $N_{c}$ (see blue points in Fig. 1). To construct $S_{\text {best }}$, we applied an iterative procedure that selects a series $\Phi^{(1)}, \Phi^{(2)}, \ldots$ of polynomial-degree combinations with $N_{c}^{(1)}<N_{c}^{(2)}<\cdots$ and $\left.\langle W\rangle^{(1)}\right\rangle$ $\langle W\rangle^{(2)}>\cdots$ (see black points in Fig. 1). We start by setting $\Phi^{(0)}=\sum_{i} \Phi_{0}\left(T_{e}\right)$ as the constant potential with $\langle W\rangle^{(0)}=1$ and $N_{c}^{(0)}=0$. On each step $k=1,2, \ldots$, we select $\Phi^{(k)}$ such that it maximizes the error reduction per added coefficients,

$$
\frac{\Delta\langle W\rangle}{\Delta N_{c}}=\frac{\langle W\rangle^{(k-1)}-\langle W\rangle}{N_{c}-N_{c}^{(k-1)}},
$$


among all polynomial-degree combinations with $N_{c}>$ $N_{c}^{(k-1)}$. We continue the iterations until step $k$ for which $\Phi^{(k)}$ reaches the upper degree limits. $S_{\text {best }}$ contains not only the elements of the series $\Phi^{(1)}, \Phi^{(2)}, \ldots$ but also all polynomial-degree combinations that exhibit a $10 \%$ lower value of $\Delta\langle W\rangle / \Delta N_{c}$ than $\Phi^{(k)}$ (see light blue points in Fig. 1). In this way, we developed a self-learning method that constructs the small subset $S_{\text {best }}$ containing optimally adjusted polynomial-degree combinations for the following detailed analysis to select the final interatomic potential.

Within the members of $S_{\text {best }}$, a further decrease of $\langle W\rangle$ is quite low for $N_{c}>50$ (see Fig. 1). Thus, we analyzed only the members with $N_{c} \leq 50$. For those polynomial-degree combinations of $S_{\text {best }}$, we constructed the corresponding interatomic potential at each of the 11 studied $T_{e}$ 's by determining the related optimal coefficients and cutoff radii that minimize $W\left(T_{e}\right)$. Using these interatomic potentials, we checked the description of the following physical properties at all studied $T_{e}$ 's: (i) the phonon band structure of the bulk diamondlike crystal structure, (ii) the cohesive energy curves of the diamondlike, fcc, bcc, and simple cubic (Sc) bulk crystal structures, and (iii) the time evolution of the atomic root-mean-square displacements (RMSDs) in the MD simulation of bulk Si. The finally selected polynomial-degree combination with $N_{c}=23$ is the one for which the corresponding interatomic potential best describes the abovementioned physical properties with a minimal possible $N_{c}$. Note that the performance of the studied members of $S_{\text {best }}$ varies considerably; i.e., not all members of $S_{\text {best }}$ represent reliable interatomic potentials. In addition, the analysis and selection, which we performed manually, can also be done fully automated.

Since the resulting optimal cutoff radii vary insignificantly around $r_{c}^{(2)}=0.63 \mathrm{~nm}, r_{c}^{(3)}=0.42 \mathrm{~nm}$, and $r_{c}^{(\rho)}=0.48 \mathrm{~nm}$ at

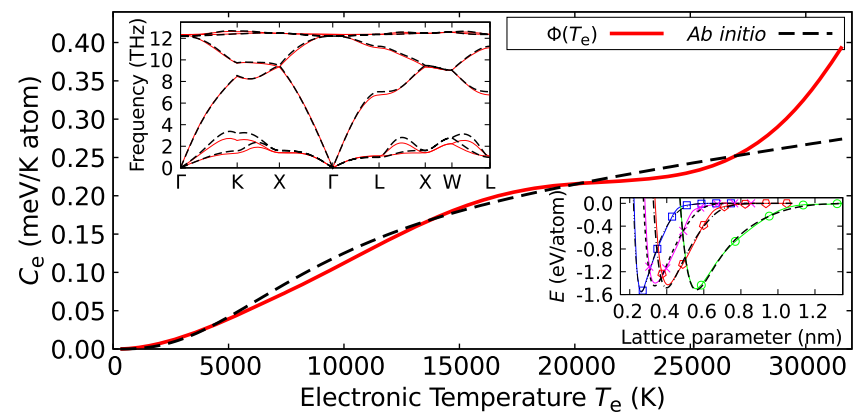

FIG. 2. Specific heat of the electrons $C_{e}$ as a function of $T_{e}$ for diamondlike bulk $\mathrm{Si}$. The upper inset indicates the phonon band structure of diamondlike bulk $\mathrm{Si}$ at $T_{e}=18315 \mathrm{~K}$. The lower inset shows the Helmholtz free cohesive energy $E$ of the diamondlike (red diamonds), fcc (magenta crosses), bcc (green circles), and Sc (blue squares) structures as a function of the lattice parameter at $T_{e}=18315 \mathrm{~K}$. Black dashed curves represent $a b$ initio data obtained using the code CHIVES and colored solid curves represent the values obtained from $\Phi\left(T_{e}\right)$. the 11 studied $T_{e}$ 's, we chose these values and kept them constant for all $T_{e}$ 's. Thus, as a result of the optimization procedure, now only the coefficients depend on $T_{e}$. These coefficients, up to now found for $11 T_{e}$ 's, were approximated by polynomials of degree 5 in $T_{e}$ to obtain a continuous dependence on $T_{e}$. This allows us to calculate the internal energy $U_{e}$ and the specific heat $C_{e}$ of the electrons using the thermodynamic relations

$$
U_{e}=\Phi-T_{e} \frac{\partial \Phi}{\partial T_{e}}, \quad C_{e}=-T_{e} \frac{\partial^{2} \Phi}{\partial T_{e}^{2}}
$$

Note that for the resulting final $\Phi\left(T_{e}\right)$, which is provided as a table and a FORTRAN subroutine in the Supplemental Material [28], both $U_{e}$ and $C_{e}$ are in very good agreement with the corresponding $a b$ initio results for bulk Si (see Fig. 2 for $C_{e}$ and Supplemental Material [28] for $U_{e}$ ). Only at $T_{e}$ 's above $27000 \mathrm{~K}, C_{e}$ of $\Phi\left(T_{e}\right)$ starts to deviate from the $a b$ initio values. It is remarkable that $\Phi\left(T_{e}\right)$ does not only reproduce lattice properties but also electronic properties. As expected, $\Phi\left(T_{e}\right)$ describes well the physical properties (i)-(iii) used for selecting the final polynomialdegree combination (see exemplary Fig. 3 and the insets of Fig. 2). The relative error of $\Phi\left(T_{e}\right)$ in the forces [second term of Eq. (6)] decreases from $26 \%$ to $6 \%$ when $T_{e}$ increases from 316 to $31577 \mathrm{~K}$. This indicates that the PES becomes less complex for higher $T_{e}$ 's. The relative error in the Helmholtz free cohesive energies [first term of Eq. (6)] always lies below $1.7 \%$. It is important to stress here that $\Phi\left(T_{e}\right)$ also reproduces forces in independent ab initio MD simulations, which were not used for its development, with the same accuracy [28]. Furthermore, the atomic RMSD perpendicular to the surface of the thin film after laser excitation is well reproduced by $\Phi\left(T_{e}\right)$ at all $T_{e}$ 's, indicating that $\Phi\left(T_{e}\right)$ accounts for surface effects [28]. The selected potential $\Phi\left(T_{e}\right)$ also reproduces properties that were not used in the fitting procedure, like the elastic

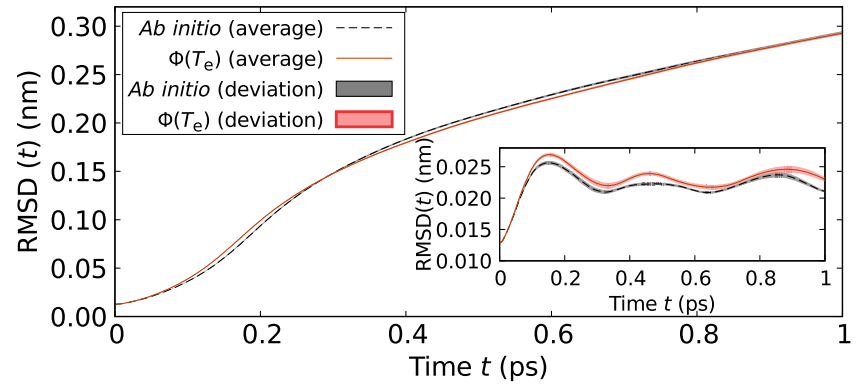

FIG. 3. Atomic root-mean-square displacements (RMSDs) during nonthermal melting [17] for bulk $\mathrm{Si}$ at $T_{e}=22104 \mathrm{~K}$ from MD simulations with 288 atoms performed $a b$ initio (black dashed curve) and using $\Phi\left(T_{e}\right)$ (red solid line) averaged over 40 runs. The inset shows the RMSD during thermal phonon squeezing [9] at $T_{e}=15789 \mathrm{~K}$ averaged over 10 runs with 640 bulk $\mathrm{Si}$ atoms. 
constants, pair-correlation function, and bond-angle distribution for finite $T_{e}$ 's in diamondlike bulk Si [28].

By performing liquid-crystal coexistence MD simulations for $65536 \mathrm{Si}$ atoms with $\Phi\left(T_{e}\right)$, we determined the melting temperature $\quad T_{m}(p)=(1199 \pm 2) \mathrm{K}-(40 \pm 3) \mathrm{KGPa}^{-1}(p)$ near zero pressure $p$. It agrees with $T_{m}(p)=(1300 \pm$ 50) $\mathrm{K}-58 \mathrm{~K} \mathrm{GPa}^{-1}(p)$ from LDA DFT [40] and differs from the experimental value, $T_{m}=(1687 \pm 5) \mathrm{K}-$ $58 \mathrm{~K} \mathrm{GPa}^{-1}(p)[41,42]$. However, and thanks to the simple physical analytical form of $\Phi\left(T_{e}\right)$, one can adjust the coefficients to reproduce the experimental $T_{m}$ without significant influences on the other properties [43]. Note that such adjustment would be impossible in machine-learning potentials [44-47], since they do not use a physically motivated and easily controllable analytical form and which, so far, do not take $T_{e}$ into account.

Notice also that previously developed potentials for $\mathrm{Si}$ at high $T_{e}$ 's $[19,23,48]$ exhibit a very inaccurate description of the atomic RMSD during thermal phonon squeezing and nonthermal melting in bulk $\mathrm{Si}$ and of the atomic RMSD perpendicular to the surface of the thin film [49]. It is important to point out that interatomic potentials fitted to data in the film geometry can describe bulk properties, whereas the opposite is not true. We have confirmed this by using our method.

To provide an application of $\Phi\left(T_{e}\right)$, we used it to perform MD simulations of a laser-excited Si nanosphere with a radius of $4.75 \mathrm{~nm}$ containing 23976 atoms, which cannot be done with $a b$ initio methods. Our simulations show that at high $T_{e}$ nonthermal melting occurs, which strongly damps the laser-excited breathing mode of the nanosphere (see Fig. S13 and Fig. S14 in the Supplemental Material [28]).

In summary, we presented an automatized method for developing highly accurate analytical $T_{e}$-dependent interatomic potentials from $a b$ initio MD simulations. We applied the method to $\mathrm{Si}$ and obtained an interatomic potential $\Phi\left(T_{e}\right)$ that can describe the material for a wide range of $T_{e}$ 's. $\Phi\left(T_{e}\right)$ is able to accurately describe laserdriven effects caused by bond softening, including nonthermal melting and thermal phonon squeezing. It is remarkable that $\Phi\left(T_{e}\right)$ does not only reproduce bulk lattice properties but also takes into account surface effects and describes electronic properties. $\Phi\left(T_{e}\right)$ can be used to describe laser processing of $\mathrm{Si}$ in the framework of the combined two temperature model MD [50] and Si subject to ion bombardment at moderate energies, for example.

The authors acknowledge the contribution by Dmitry S. Ivanov to the computer code utilized for classical MD simulations of the Si nanosphere. This work was supported by the DFG through the grant GA 465/18-1. B. B. acknowledges the support by the "Promotionsstipendium des OttoBraun Fonds" and by the "Abschlussstipendium der Universität Kassel." Computations were performed on the Lichtenberg High Performance Computer (HHLR) TU Darmstadt, on the IT Servicecenter (ITS) University of Kassel, and on the computing cluster FUCHS University of Frankfurt.

*bauerhenne@uni-kassel.de

[1] P. Stampfli and K. H. Bennemann, Theory for the instability of the diamond structure of si, ge, and c induced by a dense electron-hole plasma, Phys. Rev. B 42, 7163 (1990).

[2] V. Recoules, J. Clérouin, G. Zérah, P. M. Anglade, and S. Mazevet, Effect of Intense Laser Irradiation on the Lattice Stability of Semiconductors and Metals, Phys. Rev. Lett. 96, 055503 (2006).

[3] N. S. Grigoryan, T. Zier, M. E. Garcia, and E. S. Zijlstra, Ultrafast structural phenomena: theory of phonon frequency changes and simulations with code for highly excited valence electron systems, J. Opt. Soc. Am. B 31, C22 (2014).

[4] D. M. Fritz et al., Ultrafast bond softening in bismuth: Mapping a solid's interatomic potential with x-rays, Science 315, 633 (2007).

[5] A. Cavalleri, C. Tóth, C. W. Siders, J. A. Squier, F. Ráksi, P. Forget, and J. C. Kieffer, Femtosecond Structural Dynamics in $\mathrm{VO}_{2}$ During an Ultrafast Solid-Solid Phase Transition, Phys. Rev. Lett. 87, 237401 (2001).

[6] G. Sciaini, M. Harb, S. G. Kruglik, T. Payer, C. T. Hebeisen, F.-J. M. z. Heringdorf, M. Yamaguchi, M. H.-v. Hoegen, R. Ernstorfer, and R. J. D. Miller, Electronic acceleration of atomic motions and disordering in bismuth, Nature (London) 458, 56 (2009).

[7] M. Buzzi, M. Först, R. Mankowsky, and A. Cavalleri, Probing dynamics in quantum materials with femtosecond X-rays, Nat. Rev. Mater. 3, 299 (2018).

[8] S. L. Johnson, P. Beaud, E. Vorobeva, C. J. Milne, E. D. Murray, S. Fahy, and G. Ingold, Directly Observing Squeezed Phonon States with Femtosecond X-ray Diffraction, Phys. Rev. Lett. 102, 175503 (2009).

[9] E.S. Zijlstra, A. Kalitsov, T. Zier, and M. E. Garcia, Squeezed Thermal Phonons Precurse Nonthermal Melting of Silicon as a Function of Fluence, Phys. Rev. X 3, 011005 (2013).

[10] T. K. Cheng, J. Vidal, H. J. Zeiger, G. Dresselhaus, M. S. Dresselhaus, and E. P. Ippen, Mechanism for displacive excitation of coherent phonons in $\mathrm{Sb}, \mathrm{Bi}, \mathrm{Te}$, and $\mathrm{Ti}_{2} \mathrm{O}_{3}$, Appl. Phys. Lett. 59, 1923 (1991).

[11] M. Hase, M. Kitajima, A. M. Constantinescu, and H. Petek, The birth of a quasiparticle in silicon observed in timefrequency space, Nature (London) 426, 51 (2003).

[12] J. A. V. Vechten, R. Tsu, and F. W. Saris, Nonthermal pulsed laser annealing of Si; plasma annealing, Phys. Lett. 74A, 422 (1979).

[13] J. Reif, F. Costache, and M. Bestehorn, Self-organized surface nano-structuring by femtosecond laser processing, in Recent Advances in Laser Processing of Materials, edited by J. Periere, E. Millon, and E. Fogarassy (Elsevier, Amsterdam, 2006), p. 275.

[14] J. Reif, O. Varlamova, S. Varlamov, and M. Bestehorn, The role of asymmetric excitation in self-organized 
nanostructure formation upon femtosecond laser ablation, Appl. Phys. A 104, 969 (2011).

[15] O. Varlamova, J. Reif, S. Varlamov, and M. Bestehorn, Selforganized surface patterns originating from laser-induced instability, in Progress in Nonlinear Nano-Optics, edited by S. Sakabe, C. Lienau, and R. Grunwald (Springer, Amsterdam, 2015), Vol. 2, pp. 3-29.

[16] P. L. Silvestrelli, A. Alavi, M. Parrinello, and D. Frenkel, Ab initio Molecular Dynamics Simulation of Laser Melting of Silicon, Phys. Rev. Lett. 77, 3149 (1996).

[17] E. S. Zijlstra, A. Kalitsov, T. Zier, and M. E. Garcia, Fractional diffusion in silicon, Adv. Mater. 25, 5605 (2013).

[18] S. Khakshouri, D. Alfè, and D. M. Duffy, Development of an electron-temperature-dependent interatomic potential for molecular dynamics simulation of tungsten under electronic excitation, Phys. Rev. B 78, 224304 (2008).

[19] L. Shokeen and P. K. Schelling, Thermodynamics and kinetics of silicon under conditions of strong electronic excitation, J. Appl. Phys. 109, 073503 (2011).

[20] J. A. Moriarty, R. Q. Hood, and L. H. Yang, QuantumMechanical Interatomic Potentials with Electron Temperature for Strong-Coupling Transition Metals, Phys. Rev. Lett. 108, 036401 (2012).

[21] G. E. Norman, S. V. Starikov, and V. V. Stegailov, Atomistic simulation of laser ablation of gold: Effect of pressure relaxation, J. Exp. Theor. Phys. 114, 792 (2012).

[22] S. T. Murphy, S. L. Daraszewicz, Y. Giret, M. Watkins, A. L. Shluger, K. Tanimura, and D. M. Duffy, Dynamical simulations of an electronically induced solid-solid phase transformation in tungsten, Phys. Rev. B 92, 134110 (2015).

[23] R. Darkins, P.-W. Ma, S. T. Murphy, and D. M. Duffy, Simulating electronically driven structural changes in silicon with two-temperature molecular dynamics, Phys. Rev. B 98, 024304 (2018).

[24] P. L. Silvestrelli, A. Alavi, M. Parrinello, and D. Frenkel, Structural, dynamical, electronic, and bonding properties of laser-heated silicon: An ab initio molecular-dynamics study, Phys. Rev. B 56, 3806 (1997).

[25] T. Zier, E. S. Zijlstra, A. Kalitsov, I. Theodonis, and M. E. Garcia, Signatures of nonthermal melting, Struct. Dyn. 2, 054101 (2015).

[26] T. Zier, E. S. Zijlstra, and M. E. Garcia, QuasimomentumSpace Image for Ultrafast Melting of Silicon, Phys. Rev. Lett. 116, 153901 (2016).

[27] T. Zier, E. S. Zijlstra, S. Krylow, and M. E. Garcia, Simulations of laser-induced dynamics in free-standing thin silicon films, Appl. Phys. A 123, 625 (2017).

[28] See Supplemental Material at http://link.aps.org/ supplemental/10.1103/PhysRevLett.124.085501 for more details, which includes Refs. [29-32].

[29] H. C. Andersen, Molecular dynamics simulations at constant pressure and/or temperature, J. Chem. Phys. 72, 2384 (1980).

[30] E. Bitzek, P. Koskinen, F. Gähler, M. Moseler, and P. Gumbsch, Structural Relaxation Made Simple, Phys. Rev. Lett. 97, 170201 (2006).

[31] R. Gross and A. Marx, Festkörperphysik (Oldenburg Wissenschaftsverlag $\mathrm{GmbH}$, München, 2012).
[32] V. P. Lipp, B. Rethfeld, M. E. Garcia, and D. S. Ivanov, Atomistic-continuum modeling of short laser pulse melting of Si targets, Phys. Rev. B 90, 245306 (2014).

[33] E. S. Zijlstra, T. Zier, B. Bauerhenne, S. Krylow, P. M. Geiger, and M. E. Garcia, Femtosecond-laser-induced bond breaking and structural modifications in silicon and defective graphene: An ab initio molecular dynamics study, Appl. Phys. A 114, 1 (2014).

[34] L. Waldecker, T. Vasileiadis, R. Bertoni, R. Ernstorfer, T. Zier, F.H. Valencia, M.E. Garcia, and E.S. Zijlstra, Coherent and incoherent structural dynamics in laser-excited antimony, Phys. Rev. B 95, 054302 (2017).

[35] F. H. Stillinger and T. A. Weber, Computer simulation of local order in condensed phases of silicon, Phys. Rev. B 31, 5262 (1985).

[36] R. H. Wentorf and J. S. Kasper, Two new forms of silicon, Science 139, 338 (1963).

[37] M. S. Daw and M.I. Baskes, Semiempirical, Quantum Mechanical Calculation of Hydrogen Embrittlement in Metals, Phys. Rev. Lett. 50, 1285 (1983).

[38] V. V. Zhakhovskii, N. A. Inogamov, Y. V. Petrov, S. I. Ashitkov, and K. Nishihara, Molecular dynamics simulation of femtosecond ablation and spallation with different interatomic potentials, Appl. Surf. Sci. 255, 9592 (2009).

[39] F. Ercolessi and J. B. Adams, Interatomic potentials from first-principles calculations: The force-matching method, Europhys. Lett. 26, 583 (1994).

[40] D. Alfè and M. J. Gillan, Exchange-correlation energy and the phase diagram of Si, Phys. Rev. B 68, 205212 (2003).

[41] K. Yamaguchi and K. Itagaki, Measurement of high temperature heat content of silicon by drop calorimetry, J. Therm. Anal. Calorim. 69, 1059 (2002).

[42] A. Jayaraman, W. Klement, and G. C. Kennedy, Melting and polymorphism at high pressures in some group IV elements and III-V compounds with the diamond/zincblende structure, Phys. Rev. 130, 540 (1963).

[43] This adjustment is out of the scope of the present Letter and will be the subject of a further publication.

[44] M. Rupp, Machine learning for quantum mechanics in a nutshell, Int. J. Quantum Chem. 115, 1058 (2015).

[45] J. Behler, Perspective: Machine learning potentials for atomistic simulations, J. Chem. Phys. 145, 170901 (2016).

[46] A. Grisafi, D. M. Wilkins, G. Csányi, and M. Ceriotti, Symmetry-Adapted Machine Learning for Tensorial Properties Of Atomistic Systems, Phys. Rev. Lett. 120, 036002 (2018).

[47] A. P. Bartók, J. Kermode, N. Bernstein, and G. Csányi, Machine Learning a General-Purpose Interatomic Potential for Silicon, Phys. Rev. X 8, 041048 (2018).

[48] L. Shokeen and P. K. Schelling, An empirical potential for silicon under conditions of strong electronic excitation, Appl. Phys. Lett. 97, 151907 (2010).

[49] B. Bauerhenne and M. E. Garcia, Performance of state-ofthe-art force fields for atomistic simulations of silicon at high electronic temperatures, Eur. Phys. J. Spec. Top. 227, 1615 (2019).

[50] D. S. Ivanov and L. V. Zhigilei, Combined atomistic-continuum modeling of short-pulse laser melting and disintegration of metal films, Phys. Rev. B 68, 064114 (2003). 